\title{
Human endometrial stromal cells inhibit the pro-angiogenic stimulus of hCG in vitro
}

\author{
Marcia Riboldi ${ }^{1,+}$, Ivonne Nazir ${ }^{2}$, Belén Jara $\mathbb{1}^{2}$, Felipe Argandoña ${ }^{2}$, Cecilia Valencia ${ }^{2, \neq}$, \\ Paulo C Serafini ${ }^{1,3}$, Eduardo Leme Alves Motta ${ }^{1,4}$, Denisse Mena-Silva ${ }^{2}$, \\ Reinaldo González-Ramos², M Cecilia Johnson², Ariel Fuentes², Karina Sequeira ${ }^{2}$ and \\ Alejandro Tapia-Pizarro ${ }^{2}$ \\ ${ }^{1}$ Huntington Medicina Reprodutiva, São Paulo, Brasil, ${ }^{2} I D I M I$, Facultad de Medicina, Universidad de Chile, Santiago, \\ Chile, ${ }^{3}$ Disciplina de Ginecologia, Departamento de Obstetrícia e Ginecologia, Faculdade de Medicina, \\ Universidade de São Paulo (FMUSP), São Paulo, Brazil and ${ }^{4}$ Disciplina de Ginecologia Endocrinológica, \\ Departamento de Ginecologia, Escola Paulista de Medicina da Universidade Federal de São Paulo (UNIFSEP-EPM), \\ São Paulo, Brazil
}

Correspondence should be addressed to A Tapia-Pizarro; Email: atapiap@gmail.com

${ }^{\dagger}$ (M Riboldi is now at Igenomix, São Paulo, Brasil)

${ }^{\ddagger}$ (C Valencia is now at Laboratorio de Reproducción, Centro de Biotecnología de La Reproducción (CEBIOR-BIOREN), Universidad de La Frontera, Temuco, Chile)

\begin{abstract}
During embryo implantation, endometrial angiogenesis is regulated by signals originating from the endometrium itself and the developing embryo. It has been suggested that hCG may play a pro-angiogenic role; therefore, we sought to understand its regulatory role in blood vessel formation in human endometrium using in vivo and in vitro models. In the in vivo model, we screened 16 angiogenesis-related transcripts in the endometrium upon intrauterine administration of hCG. Oocyte donors were recruited and during their controlled ovarian stimulation cycle received a single dose of hCG or vehicle on the day of oocyte pick up during a cycle of ovarian stimulation. One hour before obtaining an endometrial sample, women received an intrauterine administration of vehicle or hCG (500, 1500 and $5000 \mathrm{IU})$. Transcript and protein analysis showed that MMP3 and VEGFA increased, whereas TIMP1 decreased. The in vitro analysis studied the angiogenic potential of conditioned medium (CM) from primary cultures of human endometrial stromal cells (ESC) stimulated with hCG. Using a 2D and 3D in vitro angiogenesis assays, our results indicate that CM from ESC almost completely inhibits the capillary-like structure formation in endothelial cells, overriding the pro-angiogenic effect of hCG; and this inhibition due to secreted factors present in CM specifically reduced the migration potential of endothelial cells. In conclusion, the endometrial stromal milieu seems to modulate the direct pro-angiogenic effects of hCG on endothelial cells during embryo implantation.
\end{abstract}

Reproduction (2020) $\mathbf{1 6 0} 673-684$

\section{Introduction}

The formation of new blood vasculature in the uterine endometrium is crucial in the formation of a receptive endometrium for embryo implantation, maintenance of early pregnancy, and development of the placenta. Angiogenesis is the process by which new capillary blood vessels are generated from pre-existing ones in a highly regulated fashion. For that, angiogenic endothelial cells must proliferate, produce molecules able to degrade the extracellular matrix, change their adhesive properties, migrate and differentiate in new vascular tubes. All these processes are controlled by the signals received by endothelial cells hence, the balance of inducers and inhibitors of angiogenesis within a given microenvironment will determine the blood vessel expansion dynamics (Hanahan \& Folkman 1996). One of the most studied molecules promoting angiogenesis is vascular endothelial growth factor (VEGF) which is produced by endometrial epithelial and stromal cells with maximum expression during the late secretory and premenstrual phases (Torry \& Torry 1997). During embryo implantation, the endometrial vascular expansion is regulated by a network of local mediators from the embryo and the endometrial stroma at the maternal-embryonic interface (Fazleabas et al. 2004, Minas et al. 2005, Perrier d'Hauterive et al. 2005). The endometrial stromal compartment comprises the largest proportion of the endometrium composed mainly by uterine fibroblasts and a mixed variable population 
of immune cells. The endometrial stromal cells (ESC) rapidly differentiate into decidualized cells (dESC) when stimulated by an implanting blastocyst, undergoing a morphological and biochemical reprogramming. After attachment and invasion, a steady supply of nutrients, as well as gas and metabolite exchanges, are provided through the endometrial tissue by means of an adequate blood perfusion via an extensive vascular network in the decidualizing stroma. Alterations in this process may lead to embryo implantation failure and inadequate placentation. Abnormal uterine blood supply is associated with higher perinatal morbidity and mortality caused by preeclampsia, intrauterine growth restriction or preterm delivery (Ahmed et al. 2000).

Human chorionic gonadotropin (hCG) is one of the earliest embryonic signals being already expressed in eight-cell embryos and reaching high local concentrations at the embryo-maternal interface. Aside from the more classical effects of hCG rescuing corpus luteum function, it has proposed that hCG may act as a pro-angiogenic factor based on its ability to promote vascular development through a direct effect on uterine endothelial cells (Zygmunt et al. 2002, Berndt et al. 2006). The presence of LH/hCG-R on endothelial cells of the uterine vessels has been described (Toth et al. 1994) through which hCG may induce angiogenesis (Berndt et al. 2006). Zygmunt et al. (2002) using a 3D in vitro angiogenesis assay showed that hCG exerted a pro-angiogenic effect inducing the migration and sprouting of uterine endothelial cells by signaling through the LHCG receptor (LHCGR). In addition, Berndt et al. (2006) confirmed the pro-angiogenic effects of hCG using in vivo models that included the chick chorioallantoic membrane, the matrigel plug assay and the aortic ring assay. In addition, they showed that hCG may induce angiogenesis indirectly exerting a paracrine regulation on endometrial epithelial cells (EEC) via the LHCGR, increasing their secretion of VEGF, which had additive angiogenic activities with hCG. In line with these results, intrauterine administration of hCG by microdialysis during the secretory phase acutely modulated the endometrial secretion of VEGF, whose levels increased as soon as $1 \mathrm{~h}$ after hCG infusion (Licht et al. 1998, 2001). Based on these results they suggest that the endometrial epithelium responds to the hCG produced by the implanting embryo by secreting VEGF which then stimulates angiogenesis on the endometrial sub-epithelial vessels (Berndt et al. 2006). Later on during embryo implantation, the hCG secreted by the invading embryo might stimulate angiogenesis directly on uterine endothelial cells. However, most of VEGF produced by the endometrial epithelium is secreted apically (Hornung et al. 1998) so it is unlikely that it has an angiogenic role in human endometrium. Alternatively, hCG might exert paracrine effects on endometrial stromal cells which in turn may create a pro-angiogenic microenvironment at the time of embryo implantation. Unfortunately, no data is available regarding this matter.
In the present study, we aimed at evaluating the acute in vivo regulation of angiogenic molecules by hCG in human endometrium and to determine whether the effect of hCG on endometrial stromal cells (ESC) modulate angiogenesis in vitro.

\section{Materials and methods}

\section{Patients for intrauterine hCG administration protocol}

Endometrial tissue samples $(n=25)$ were obtained from a woman undergoing controlled ovarian hyperstimulation $(\mathrm{COH})$ cycles for oocyte donation at Huntington Center of Reproductive Medicine, São Paulo, Brazil. This study was approved by Hospital e Maternidade Santa Joana's Institutional Review Board and a signed informed consent was obtained from all patients who agreed to take part. A total of 25 oocyte donors were recruited to participate in this study who were blindly randomized to either no treatment (group $\mathrm{A}, n=5$ ), vehicle (group B, $n=5$ ) or urinary hCG (Choriomon; IBSA, Lugano, Switzerland) in three doses: 500 IU (group C, $n=5$ ), 1500 IU (group D, $n=5$ ) and 5000 IU (group D, $n=5$ ) using simple randomization by the oocyte donor coordinator at Huntington Center. The doses of hCG were selected based on previous reports showing improved embryo implantation rates with the intrauterine instillation of hCG before embryo transfer (Mansour et al. 2011, Huang et al. 2017). No sample size calculations are provided due to the pilot nature of this study.

Oocyte donors consisted of healthy, fertile women (age $18-30$ years old), with a $\mathrm{BMI}<30 \mathrm{~kg} / \mathrm{m}^{2}$ who had undergone extensive screening prior to their participation as oocyte donors. Women with irregular menses, using an intrauterine device, or previously diagnosed polycystic ovary syndrome were excluded from this study. In addition, women with signs of ovarian hyperstimulation syndrome were removed from the study. All patients had normal serum FSH, LH and estradiol on day 3 beginning of the cycle. Controlled ovarian stimulation was performed in women from all groups. Briefly, after menstruation on day 3 started with r-FSH (300 IU/day; Gonal-F; Merck) plus LH (75 IU/day; Luveris; Merck) and follicular growth was monitored by transvaginal ultrasound starting on day 4 of gonadotropin administration. No significant differences were found in the total gonadotropin dosage or the length of stimulation among groups (data not shown). When adequate follicular growth and serum $\mathrm{E}_{2}$ levels were observed, final oocyte maturity was induced with $\mathrm{GnRH}$-antagonist (Cetrotide; Merck). Oocyte retrieval was performed 35 h later through transvaginal ultrasound. From each woman, one endometrial sample was obtained on the day of transvaginal oocyte pick up. For that, oocyte donors were placed in the lithotomy position and a speculum was utilized to visualize the cervix. A catheter was passed through the cervical os, allowing for intrauterine administration of $50 \mu \mathrm{L}$ IVF culture media (Global media, LifeGlobal Group, Guilford, CT, EE.UU) with or without hCG. Patients remained in lithotomy position for at least $10 \mathrm{~min}$ following infusion to prevent leakage. It has been shown that several secreted molecules are regulated after $1 \mathrm{~h}$ of continuous intrauterine administration of hCG 500 IU/ $\mathrm{mL}$ (Licht et al. 2001, 2007). Hence, $1 \mathrm{~h}$ following intrauterine injection of vehicle or hCG, an endometrial sample was 
obtained using a sterile biopsy catheter (Pipelle de Cornier, Prodimed, Neuilly-en-Thelle, France). The tissue sample in group A was obtained without intrauterine administration.

\section{Patients and endometrial sample for stromal cells isolation}

Endometrial tissue samples from mid secretory phase were obtained using a Pipelle catheter from women with regular menstrual cycles (35-50 years old) and no apparent endometrial dysfunction that were undergoing benign gynecologic surgery. Approval was obtained from the Review Board at Metropolitan Central Health Service, Ministry of Health, Government of Chile.

\section{Endometrial processing}

A portion of each sample was fixed in $4 \%$ paraformaldehyde in PBS, embedded in paraffin, and sections were stained with hematoxylin and eosin for histological examination. The remaining tissue was snap frozen in liquid nitrogen and stored at $-80^{\circ} \mathrm{C}$ until use. Histological dating of the menstrual cycle was performed for all endometrial biopsies by an experienced pathologist according to the method of Noyes et al. (1950). In samples used for ESC isolation, endometrium from midsecretory phase was confirmed.

ESCs were isolated as described before (Tapia-Pizarro et al. 2013). Briefly, the endometrial tissue was washed twice with PBS, minced in small pieces and digested for $1 \mathrm{~h}$ at $37^{\circ} \mathrm{C}$ in $0.5 \%$ collagenase type $3(45 \mathrm{U} / \mathrm{mL}$; Worthington Biochemical Corp., Lakewood, NJ, USA) and DNase I (Sigma). Then the suspension was filtered through a $100-\mu$ m nylon sieve (Falcon, BD, Franklin Lakes, NJ, USA) followed by a 40- $\mu$ m nylon sieve (Falcon). ESCs that passed the 40-mm sieve were thoroughly washed with PBS, resuspended in DMEM/F-12 (Gibco) 10\% fetal bovine serum (FBS, Gibco) and 1\% antibiotic-antimicotic (Gibco) and seeded in 75- $\mathrm{cm}^{2}$ culture flasks (Orange Scientific, Braine-l'Alleud, Belgium).

\section{Preparation of conditioned media (CM)}

CM were made from ESC, decidualized ESC (dESC), TM4 and NRK cells. TM4 cell line is derived from Sertoli cells obtained from immature mice (Mather 1980) and normal rat kidney (NRK) cell line (clone 49F) is derived from kidney fibroblasts (de Larco \& Todaro 1978). All cells were cultured in DMEM/F12 complete medium and grown until confluence. Decidualization of ESC was induced by the addition of $0.5 \mathrm{mM}$ of 8 -bromoadenosine 3',5'-cyclic monophosphate (8Br-cAMP, Sigma) and $1 \mathrm{mM}$ medroxyprogesterone acetate (MPA, Sigma) during 5 days. This medium was changed every $48 \mathrm{~h}$ and $\mathrm{CM}$ from $\mathrm{dESC}$ was prepared following decidualization without the $8 \mathrm{Br}$-CAMP and MPA. CM from all cell cultures was prepared from confluent $100 \mathrm{~mm}$ culture dishes. Cells were washed with PBS to remove growth factors and serum starved for 12-14 h. Then, culture medium was replaced with $5 \mathrm{~mL}$ of serum-free DMEM/F12. ESC and $\mathrm{dESC}$ were incubated with or without hCG (Sigma; 1, 10 or $100 \mathrm{IU} / \mathrm{mL}$ ) to prepare $\mathrm{CM}$ under hCG stimulation (CM-1, CM-10 and CM-100, respectively). The doses of hCG have been taken from previous in vitro experiments and correspond to the physiological hCG concentrations reached in the first 2 weeks of pregnancy (Berndt et al. 2006, 2009). After incubated for 72 $\mathrm{h}$, the supernatant was collected and centrifuged for $5 \mathrm{~min}$ at $3000 \mathrm{~g}$ to remove cell debris, filtered with a $0.2-\mu \mathrm{m}$ filter and stored at $-80^{\circ} \mathrm{C}$ until used. For some experiments, $\mathrm{CM}$ were concentrated 100X using Amicon Ultracel 3K Centrifugal Filters (Merck Millipore), which have a 3 kDa cutoff range for retention and then diluted 1:100 with fresh DMEM/F12 culture medium.

\section{$R N A$ isolation and real-time $q P C R$ analysis}

Total RNA was isolated from frozen tissue samples using the RNeasy mini kit with on-column DNAse I digestion (Qiagen) according to the instructions provided by the manufacturer. Total RNA $(1 \mu \mathrm{g})$ was reverse transcribed to cDNA using the SuperScript III kit (Invitrogen). Gene expression levels were assessed using the QuantiTect SYBR Green PCR Kit (Qiagen) in a StepOne Real-Time PCR thermocyler (Applied Biosystems). Glyceraldehyde 3-phosphate dehydrogenase gene (GAPDH) was used for normalization and the references for the primer pairs used for amplification of the 16 analyzed transcripts are given in Supplementary Table 1 (see section on supplementary materials given at the end of this article).

\section{Protein extraction and immunoblot detection}

Total protein was obtained with acetone precipitation from buffer RLT lysates as indicated by the supplementary protocol from Qiagen. Briefly, after centrifuging the tissue lysate through the RNasy spin columns, the flow-through was recovered and four volumes of cold acetone was added and incubated for $30 \mathrm{~min}$ at $-20^{\circ} \mathrm{C}$. Then the precipitate was pelleted by centrifuging for $10 \mathrm{~min}$ at maximum speed in an Eppendorf 5414 benchtop centrifuge (Eppendorf, Hamburg, Germany). The supernatant was discarded and the pellet was washed twice with ethanol, air-dried and resuspended in ProteoJET lysis buffer (Fermentas AB, Vilnius, Lithuania) supplemented with phosphatase inhibitors and a cocktail of protease inhibitors (Sigma). Protein concentration was determined using the bicinchoninic acid method (Thermo Scientific) and $25 \mu \mathrm{g}$ were separated by $8 \%$ of sodium dodecyl sulfate PAGE (SDSPAGE) under reducing conditions, and then transferred onto polyvinylidene difluoride membrane (Hybond, Amersham). The membranes were blocked with $5 \%$ fat-free milk for 30 min, and then immunoblotted with primary antibodies against MMP-3 (IM36L, Oncogene Research Products, Boston, MA, USA), VEGF (sc-7269, Santa Cruz Biotechnologies) or TIMP-1 (MAB3301; EMD Millipore) overnight at $4^{\circ} \mathrm{C}$. The bound antibodies on the membrane were detected using appropriate peroxidase-coupled secondary antibodies for $1 \mathrm{~h}$. Beta-actin was detected in the same blots that were stripped and re-probed. Immunodetection was developed using chemiluminescence (ECL, Thermo; Indianapolis, IN, USA) and images were captured with the Discovery team $10 \mathrm{gD}$ (Ultralum, Claremont, CA, USA). The ratio of relative intensities for the specific bands vs the obtained for beta-actin was quantitated by optical densitometry of the bands using the Carestream MI5.0.6.20 software (Carestream Health, Rochester, NY, USA). 


\section{Endometrial immunostaining}

Immunohistochemical staining was performed for immunolocalization of VEGFA, TIMP1 and MMP3. Paraformaldehyde-fixed paraffin embedded tissue was sectioned at $5 \mu \mathrm{m}$ and placed on microscope slides. Each tissue section was dewaxed in xylene and re-hydrated via graded alcohols. Heat-induced epitope retrieval was performed using citrate buffer followed by incubation with $0.3 \%$ hydrogen peroxide in methanol (20 min, room temperature) for quenching the endogenous peroxidase activity. Slides were blocked for $1 \mathrm{~h}$ in 10\% mouse IgG1 (Dako) and then incubated overnight at $4{ }^{\circ} \mathrm{C}$ in one of the following primary antibodies raised against: VEGFA (sc-365578, Santa Cruz Biotechnologies); TIMP1 (MAB3301; EMD Millipore) or MM-3, (Oncogene Research Products). Subsequently, sections were incubated in respective biotinylated secondary antibodies for anti-mouse, anti-rabbit or anti-goat (BA-9200, BA-1000, BA-9500; Vector Laboratories, Burlingame, CA, USA) followed by HRP-conjugated streptavidin. Diaminobenzidine (SK-4100, DAB Substrate Kit, Vector Laboratories) was used as chromogen for immunoreactivity producing brown staining.

\section{Analysis of angiogenesis-related molecules in CM}

The Human Angiogenesis Antibody Array (R\&D systems) was used for the screening of 55 angiogenesis-related molecules according to the instructions from the manufacturer. Pooled CM were prepared from ESC and dESC ( $n=3$ per condition) stimulated with vehicle or $100 \mathrm{IU} / \mathrm{mL}$ hCG and assayed in the membrane-based sandwich immunoassay. Briefly, the array membranes (spotted with capture antibodies to specific target proteins) were blocked with blocking solution for $1 \mathrm{~h}$ and then incubated with a mixture of $\mathrm{CM}$ and a cocktail of biotinylated detection antibodies overnight at $4{ }^{\circ} \mathrm{C}$ on a rocking platform. Following a wash step to remove unbound molecules, streptavidin-horseradish and chemiluminiscent detection reagents were added sequentially to visualize the captured proteins. All experiments were performed in duplicate. The data on the photodocumented images from the membranes were quantified by analyzing the pixel density using ImageJ analysis software (http://rsbweb.nih.gov/ij/). Average signal (pixel density) of the pair of duplicate spots representing each protein was determined and the average background signal was subtracted. The corresponding protein signals were compared between the membranes by normalizing the intensity data based on the pixel intensity of the positive control spots. All experiments were performed in duplicate. Changes of $>20 \%$ of variation for up or downregulation was considered for further analyses.

\section{Casein zymogram}

Enzymatic activity corresponding to caseinolytic activities of 57-kDa MMP3 was detected in equal volumes of individual samples from CM in duplicate as described before (TapiaPizarro et al. 2013). Briefly, the medium samples were treated with sample buffer without boiling or reduction. Sodium dodecyl sulfate-PAGE (SDS-PAGE) was performed using a $10 \%$ gel containing $2 \mathrm{mg} / \mathrm{mL}$ casein (Sigma) at $100 \mathrm{~V}$ for $90 \mathrm{~min}$ at $4^{\circ} \mathrm{C}$. The gels were soaked in $2.5 \%$ Triton $\mathrm{X}-100$
(Sigma) for $30 \mathrm{~min}$ at RT to remove the SDS and incubated in a reaction buffer at $37^{\circ} \mathrm{C}$ overnight to allow proteinase digestion of its substrate. Gels were rinsed again in distilled water, stained with $0.5 \%$ Coomassie brilliant blue R-250 in $40 \%$ methanol and $10 \%$ acetic acid for $2 \mathrm{~h}$, and destained with $40 \%$ methanol and $10 \%$ acetic acid. Proteolytic activities appeared as clear bands of lysis against a blue background of stained casein. Bands were identified by molecular weight using a molecular weight marker (Bio-Rad Laboratories). To verify that the detected caseinolytic activity was specific MMP-3, the gels were treated in parallel experiments with reaction buffer containing $10 \mathrm{mM}$ EDTA. Enzymatic activity was visualized by negative staining and semiquantified by densitometric analysis of zymograms using the Image-Pro Plus Software (Media Cybernetics, Inc., Silver Spring, MA, USA). The mean of the controls was set to one, and the data are expressed as change from control values.

\section{Enzyme-linked immunosorbent assay (ELISA)}

ELISAs were performed on CM to confirm the semiquantitative results for VEGF and TIMP1 obtained with the antibody array. For that, commercial ELISA kits (R\&D Systems) were used according to the manufacturers' instructions. CM were thawed and concentrated 10-fold using a vacuum dryer prior to performing the assay for each individual sample in duplicate. The lower detection limit of the assay for VEGFA and TIMP1 was $9 \mathrm{pg} / \mathrm{mL}$ and $0.08 \mathrm{ng} / \mathrm{mL}$, respectively. The intraassay coefficient of variation was $2.4-10.2 \%$; the interassay coefficient of variation was $5.1-8.7 \%$. Immunoreactive VEGFA and TIMP1 was quantitatively measured in CM derived from ESC of at least six different donors.

\section{Cell viability assay}

The viability of EA.hy926 cells after incubation with CM or treatments was determined using the MTS colorimetric assay CellTiter 96 AQ assay (Promega Corp.). Briefly, cells were plated in 96-well flat bottom tissue culture plates at a concentration of $1 \times 10^{4}$ cells/100 $\mu \mathrm{L} /$ well with DMEM/F12 at $37^{\circ} \mathrm{C}, 5 \% \mathrm{CO}_{2}$. The cells were allowed to grow for $24 \mathrm{~h}$, and then the culture medium was removed and washed twice with PBS. CM or treatments was added to each well $(100 \mu \mathrm{L})$. After the cells were incubated for $72 \mathrm{~h}$, the medium was removed and washed twice with PBS. WST-1 reagent was added to the wells and incubated at $37^{\circ} \mathrm{C}$ for $2 \mathrm{~h}$. The reaction solution was transferred to a new 96-well plate in sextuplicate, and the absorbance at 490-nm was measured using a microplate reader (Bio-Tek Instruments). The assays were performed at least four times with different batches of ESC- or dESC-conditioned medium. In control studies, the cells were re-fed with DMEM containing $0.1 \% \mathrm{BSA}, 1 \% \mathrm{FBS}$, antibiotics, and $10 \mathrm{IU} / \mathrm{mL}$ hCG or VEGF (sc-4570, 10 ng/mL; Santa Cruz Biotechnologies)

\section{In vitro analysis of $2 D$ angiogenic activity}

Angiogenic activity was determined in CM obtained from cell cultures, evaluating the development of capillary-like structures in cultures of the endothelial-like cell line EA.hy926 as described 
by Aranda and Owen (2009). EA.hy926 cells have maintained the phenotype of endothelial cells and were obtained by hybridization of human umbilical vein endothelial cells with the A549/8 human lung carcinoma cell line. Forty-eight-well plates were coated with $150 \mu \mathrm{L}$ of Matrigel (BD Biosciences, Franklin Lakes, NJ, USA) and incubated for $30 \mathrm{~min}$ at $37^{\circ} \mathrm{C}$. To investigate the effects of CM on formation of capillary-like structures, 40,000 cells were seeded onto Matrigel-coated wells in the presence of $500 \mu \mathrm{L}$ of the different media under investigation. After incubation for $8 \mathrm{~h}$, ten representative images per well were obtained at $40 \times$ magnification using a TMS Inverted Phase Contrast Microscope (Nikon). Images were analyzed to calculate an in vitro angiogenic score.

\section{In vitro analysis of $3 D$ angiogenic activity}

CM were analyzed for its angiogenic activity in a 3D assay model, according to Zygmunt et al. (2002) with some modifications. Cytodex 3 microcarriers (CDX, Sigma) were combined with EA.hy926 cells to a final concentration of $\sim 30$ cells/CDX and shaken gently every $20 \mathrm{~min}$ for $4 \mathrm{~h}$ at $37^{\circ}$ $\mathrm{C}$ and $5 \% \mathrm{CO}_{2}$. The coated CDXs were cultivated in IMDM culture medium (GIBCO) at $37 \mathrm{C}^{\circ}, 5 \% \mathrm{CO}_{2}$ for $1-2$ days before they were embedded into a fibrin gel. Fibrinogen $(2.5 \mathrm{mg} / \mathrm{mL}$, Sigma) with 0.15 units $/ \mathrm{mL}$ of aprotinin (Sigma) was dissolved in PBS ( $\mathrm{pH} 7.1 ; 1.5-2.5 \mathrm{mg} / \mathrm{mL}$ ) and filled into a 12-well plate (0.6 mL/well). Cell-coated CDXs ( 100 CDX/well) and thrombin $(0.65 \mathrm{U} / \mathrm{mL}$; Sigma) were added for inducing fibrin polymerization. Fibrinogen/bead solution was allowed to clot for $5 \mathrm{~min}$ at room temperature and then at $37^{\circ} \mathrm{C}$ and $5 \% \mathrm{CO}_{2}$ for $20 \mathrm{~min}$. One milliliter of DMEM/F12 was added to each well and equilibrated with the fibrin clot for $30 \mathrm{~min}$ at $37^{\circ} \mathrm{C}$ and $5 \% \mathrm{CO}_{2}$. DMEM/F12 was removed from the wells and replaced with $1 \mathrm{~mL}$ of $\mathrm{CM}$. Medium was changed every other day. VEGFA and hCG were used the indicated concentrations. For quantification of in vitro angiogenic activity, high-resolution images of beads were captured at 24, 48 and 72 h using a TMS Inverted Phase Contrast Microscope (Nikon) with a $4 \times$ objective for considering all the quantified features in the depth of field in focus. In all the evaluated conditions, no lumen formation or anastomosis was observed and the number of sprouts and branching points yielded similar results. Sprout formation and branching points were quantified in eight microscope fields per well. The numbers of microcarriers, sprouts and branching points were counted, and results were expressed as the number of sprouts or branching points per cell-coated CDX. All assays were repeated at least three times, and four wells were used for each treatment condition.

\section{Scratch wound healing assay}

HUVEC were grown to confluence in 24-well plates. Then a cell-free strip was created by scratching on the midline of the culture well confluent monolayers of cells in the 24-well plates using 0.2-mL pipette tip, and CM were added. Images were captured with an inverted phase contrast light microscope (Olympus Optical Co. Ltd.) after incubation for
6 h. Serial microphotographs from three randomly selected fields were obtained. The experiments were repeated three times. The invaded area by migrated cells was calculated using the ImageJ analysis software. Cell migration was expressed as invaded area: (original scratch area - new scratch area)/ original scratch area $\times 100 \%$.

\section{Statistical analysis}

All in vitro experiments were performed using cell preparations from at least three different endometrial biopsies $(n \geq 3)$. Raw data were transformed to ratio from baseline condition. Data were analyzed using a Kruskal-Wallis test followed by the Dunn's post hoc test with Bonferroni correction; $P<0.05$ was considered to be statistically significant. Experimental data are presented as the mean, and the number of experiments is indicated in the figure legends as $n$.

\section{Results}

\section{Regulation of angiogenesis-related molecules in human endometrium in vivo}

We assessed the endometrial regulation of 16 transcripts coding for genes related with angiogenesis by intrauterine administration of hCG. Real-time qPCR analysis showed that $1 \mathrm{~h}$ after administration of hCG, transcript levels for MMP3 and VEGFA significantly increased with the doses of 500 and 5000 IU hCG, respectively (Fig. 1). Previously, we and others showed that hCG decreased TIMP

1 secretion in endometrial stromal cells in vitro (Fluhr et al. 2008, Tapia-Pizarro et al. 2013); however, neither the levels of TIMP1 mRNA nor those for the other 13 analyzed transcripts changed with the intrauterine administration of the hormone (data not shown). We selected MMP3, VEGFA and TIMP1 to study a possible acute regulation of their protein levels in the endometrium with the intrauterine instillation of hCG. Western blot analyses showed that protein levels for MMP3 and VEGFA were regulated in the same manner as their transcripts (Fig. 2A and C; and 2B and the D, respectively) which was in line with the IHC staining for MMP3 and VEGFA (Fig. 2E, F, G and H, I, J, respectively). An increased immunoreactive level for MMP3 was observed in samples stimulated with 500 IU of hCG in both epithelium and stroma but not in samples that received $5000 \mathrm{IU}$ of the gonadotrophin (Fig. 2F and G). Immunostaining for VEGFA in endometrial sections showed an increased label at the dose of $5000 \mathrm{IU}$, which was more intense in epithelium than stroma (Fig. 2)). Although TIMP1 transcript levels did not change, its protein levels assessed by immunoblot and confirmed by ELISA showed that it was acutely downregulated by hCG (Fig. 3A, B, C, D, E and F). IHC staining for TIMP1 was also concordant with the protein analyses displaying reduced immunoreactive label both in epithelium and stroma with the dose of 5000 IU (Fig. 3F). 

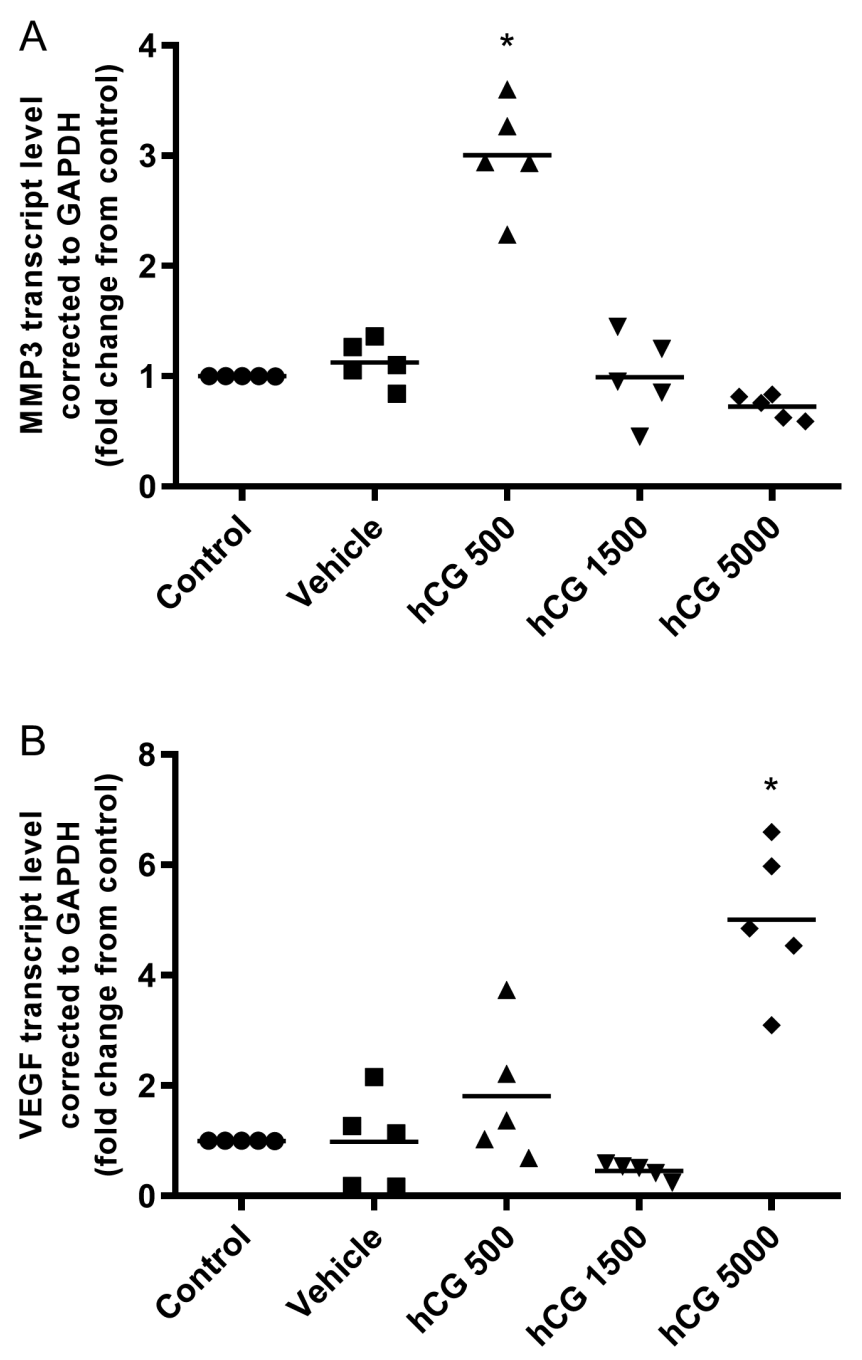

Figure 1 Intrauterine administration of hCG acutely regulated mRNA levels for MMP3 (A) and VEGFA (B) in human endometrium. hCG was administered at the time of oocyte pick-up during controlled ovarian hyperstimulation for oocyte donation. Vehicle or hCG (500, 1500 or $5000 \mathrm{IU}$ ) was administered and $1 \mathrm{~h}$ after an endometrial sample was obtained for transcripts analysis using quantitative real-time RT-PCR. Control group did not have intrauterine administrations. $n=5$ per group; ${ }^{*} P<0.05$ with respect to vehicle group.

\section{hCG stimulation of ESC or dESC in vitro do not support a clear pro- or anti-angiogenic response}

As a proxy to address the question whether hCG indeed regulates angiogenesis by modulating the secretion of angiogenesis-related factors in endometrial stroma, we screened the change in the secreted profile of 55 angiogenesis-related factors using a protein array in pooled conditioned media (CM) from endometrial stromal cells. Since decidualization may change the response of ESC to hCG, we prepared CM from ESC and in vitro decidualized ESC (dESC) stimulated with hCG or vehicle. The regulated angiogenesis-related proteins in pooled CM from ESC or dESC is presented in Supplementary Fig. 1 and Supplementary Table 2. The data from the protein-array analysis of CM showed that upon hCG stimulus, the overall responses did not exhibit a clear pro- or anti-angiogenic response in ESC and dESC. We evaluated the secreted levels of MMP3 by casein zymography and confirmed the secreted levels of VEGFA and TIMP1 by ELISA in individual samples of CM from ESC and dESC. Enzymatic activity for MMP3 did not change significantly among the evaluated CMs (Fig. $4 \mathrm{~A}$ and $\mathrm{B}$ ). In ESC-CM, TIMP1 secreted levels showed a biphasic regulation in response to increasing doses of hCG with a significant reduction of $-44.1 \%$ at $10 \mathrm{IU} / \mathrm{mL}$ while significantly increased $45 \%$ with $100 \mathrm{IU} / \mathrm{mL}$ (Fig. 4C), effect not observed in dESC-CM. No significant changes were observed in VEGFA secretion in ESC-CM (Fig. 4E); however in dESC-CM, hCG significantly reduced the released VEGFA $(-27.6 \%)$ only at the dose of $100 \mathrm{IU} / \mathrm{mL}$ (Fig. 4F).

\section{CM from ESC or dESC inhibits hCG-induced increase in angiogenic potential}

The most accepted model for angiogenesis regulation suggests that capillary growth is driven by the balance of pro- and anti-angiogenesis signaling molecules which may include many more factors than those analyzed in the protein array. To evaluate the overall effect of hCGinduced secretions in ESC and dESC on angiogenesis, we performed in vitro assays to identify direct effects on endothelial cell function. The CM from ESC and dESC was assayed in a 2D in vitro angiogenesis assay evaluating the formation of capillary-like tubes by the endothelial-like cell line EA.hy926 on a basement membrane matrix. LH/hCG receptor expression in EA.hy926 cells was confirmed by RT-qPCR and immunostaining (data not shown). CM prepared from ESC or dESC previously stimulated with vehicle or hCG at doses of 1,10 or $100 \mathrm{IU} / \mathrm{mL}(\mathrm{CM}-1,-10$ and -100 , respectively) did not show differences among their angiogenic score. Unexpectedly, CM from ESC or dESC suppressed the pro-angiogenic effect of hCG (Fig. 5A and B). Representative photomicrographs of the in vitro 2D-angiogenesis assay are shown in Supplementary Fig. 2.

To further analyze the modulating effect of ESCderived secretions on hCG-induced angiogenesis in vitro, endothelial cell spheroids provide a unique 3D approach and resemble more closely the in vivo conditions where extracellular matrix and cell-cell contacts can be mimicked (Supplementary Fig. 3). In our in vitro 3D-angiogenesis assay, among the parameters that can be evaluated, we only could consistently assess sprouts and branching-points formation. At $24 \mathrm{~h}$ of incubation, CM from ESC induced a profound reduction in number of sprouts regardless of the previous hCG stimulus or in the presence of hCG or VEGFA (Fig. 6A). At 48 and $72 \mathrm{~h}$ of incubation, the inhibitory effect of 
A

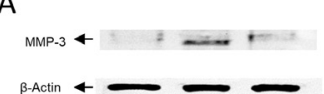

C

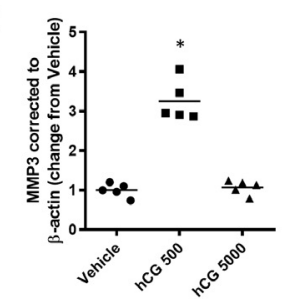

B

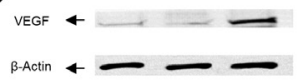

D

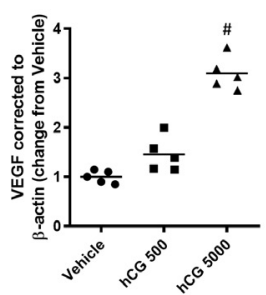

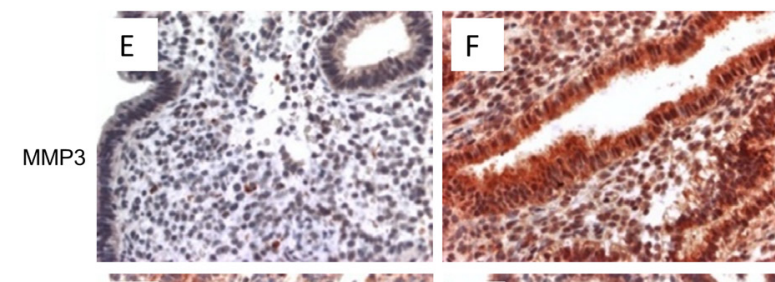

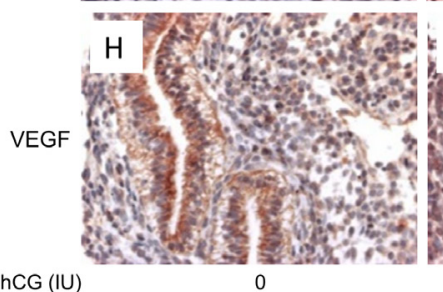

hCG (IU)
0
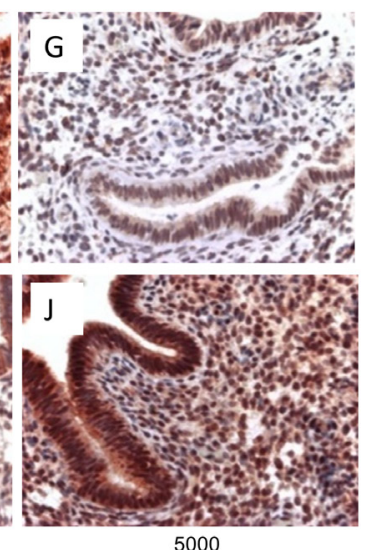

Figure 2 Intrauterine administration of hCG acutely regulated MMP3 and VEGFA in human endometrium. hCG was administered at the time of oocyte pick-up during controlled ovarian hyperstimulation for oocyte donation. Vehicle or hCG $(500,1500$ or $5000 \mathrm{IU})$ was administered and $1 \mathrm{~h}$ after an endometrial sample was obtained. Control group did not have intrauterine administrations. Total cell lysates for endometrial samples were immunoblotted with MMP3 or and VEGFA. $\beta$-Actin was used as a loading control. Representative blot for MMP3 (A) and VEGFA (B) in endometrial samples. Mean optical density of bands for MMP3 (C) and VEGFA (D) corrected to $\beta$-actin; $n=5$ per group; ${ }^{*} P<$ 0.05 with respect to vehicle and hCG 5000 groups; ${ }^{\sharp} P<0.05$ with respect to vehicle and hCG 500 IU groups. Immunohistochemical staining for MMP3 (E, F and G) and VEGFA $(H$, $I$ and $J$ ) in human endometrial tissue from women with intrauterine administration of vehicle or hCG (500 or 5000 IU, magnification, 200x).
CM was maintained except for VEGFA (Fig. 6A). The results obtained for the number of branching-points were essentially the same as for the number of sprouts (data not shown). To determine whether the modulation of hCG-induced angiogenesis by secretions from ESC was a cell-type-specific effect, we prepared CM from the cell lines NRK (derived from rat kidney fibroblasts) and TM4 (derived from mouse Sertoli cells) which were used as controls (CM-control 1 (CMC1) and CM-control
2 (CMC2), respectively). The 3D-angiogenesis assay analyzed at 24, 48 and $72 \mathrm{~h}$ of incubation showed that $\mathrm{CMC1}$ and $\mathrm{CMC2}$ did not induce the profound inhibition on angiogenesis exhibited by ESC-derived CM, suggesting a cell-type-specific effect (Fig. 6B). To rule out that the observed inhibitory effect was due to the exhaustion of nutrients and/or accumulation of metabolic waste in ESC-derived CM, we concentrated the CM 100X using Microcon centrifugal units with a 3

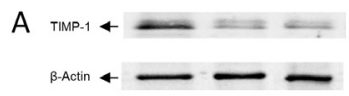

B

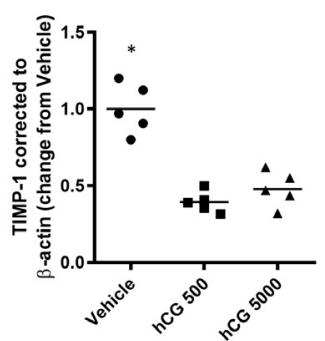

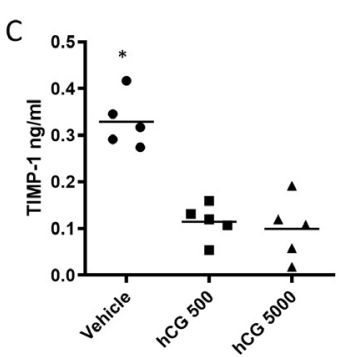

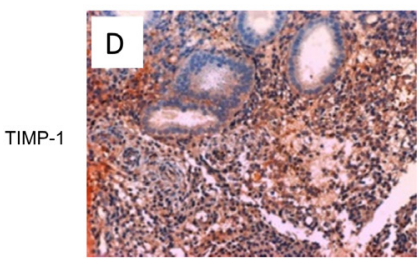

0

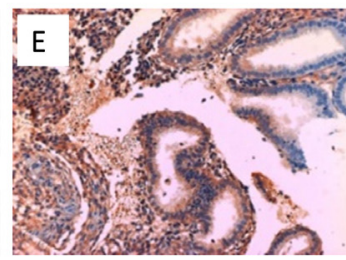

500

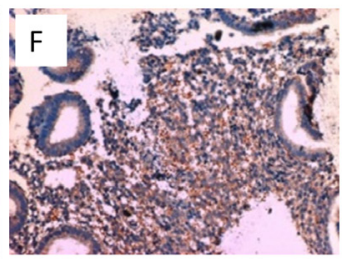

5000
Figure 3 Intrauterine administration of hCG acutely regulated TIMP1 in human endometrium. hCG was administered at the time of oocyte pick-up during controlled ovarian hyperstimulation for oocyte donation. Vehicle or hCG $(500,1500$ or $5000 \mathrm{IU})$ was administered and $1 \mathrm{~h}$ after an endometrial sample was obtained, $n=5$ per group. Total cell lysates for endometrial samples were immunoblotted with TIMP1. $\beta$-Actin was used as a loading control. (A) Representative blot for TIMP1 in endometrial samples. (B) Mean optical density of band for TIMP1 corrected to $\beta$-actin; $n=5$ per group; $* P<0.05$ with respect to hCG 500 and 5000 groups. (C) Decreased TIMP1 levels in endometrial tissue stimulated in vivo with hCG were confirmed by ELISA. Immunohistochemical staining for TIMP1 (D, E and F) in human endometrial tissue from women with intrauterine administration of vehicle or hCG (500 or 5000 IU; magnification, 100x). 

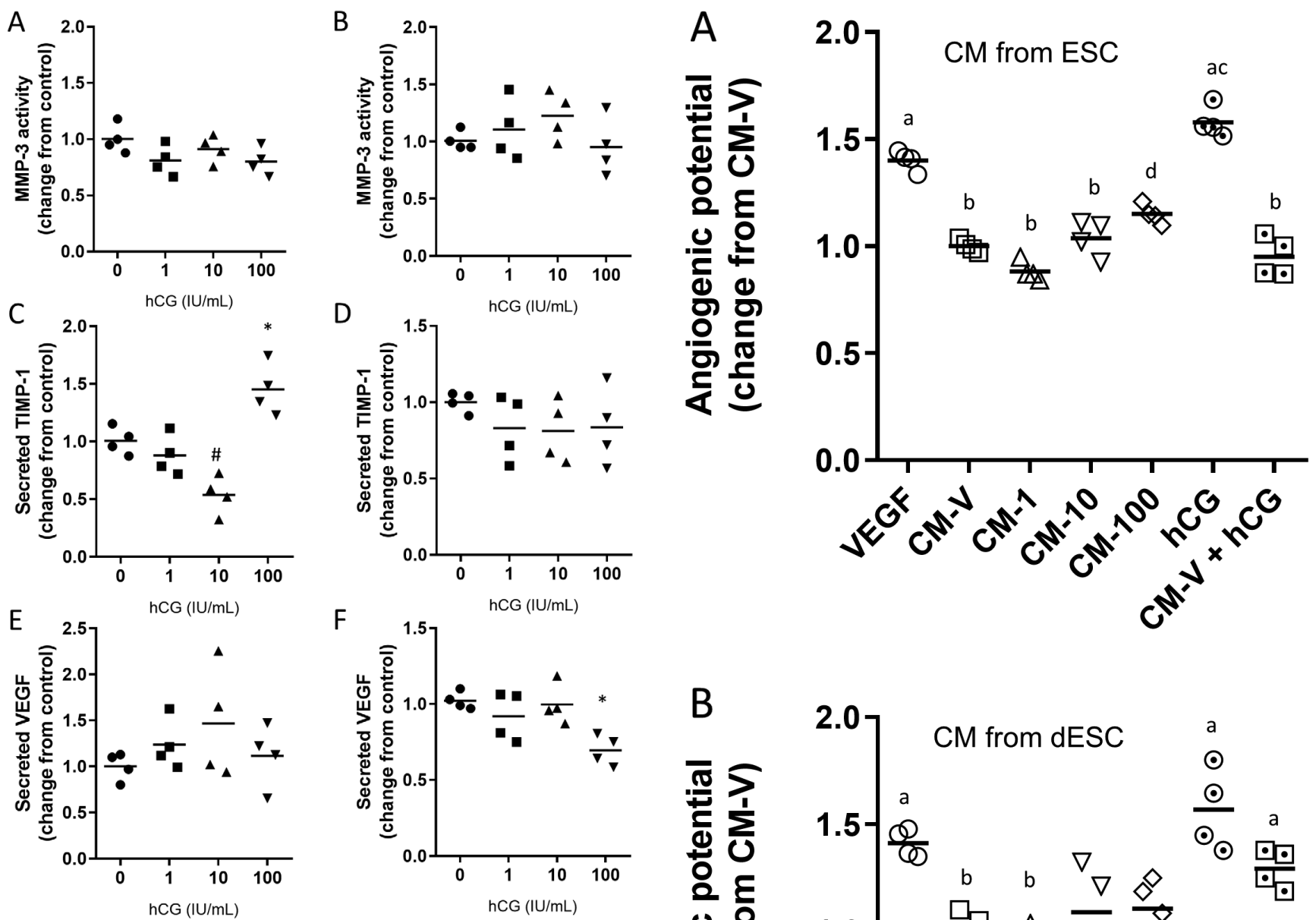

Figure 4 Effect of hCG on MMP3, TIMP1 and VEGFA secretion by human endometrial stromal cell (ESC) cultures. ESC or in vitro decidualized ESC (dESC) were stimulated with vehicle or hCG (1-100 IU/mL). Conditioned media (CM) were harvested at $72 \mathrm{~h}$ and analyzed. CM were analyzed by casein zymography followed by densitometry corresponding to proMMP3 from ESC (A) and dESC (B). Effects on TIMP1 and VEGFA secretion were confirmed by ELISA in $\operatorname{ESC}(C, D)$ and $d E S C(E, F)$, respectively. Assays were performed with $\mathrm{CM}$ from individual cultures (not pooled, $n=4$ per condition). Casein zymography and ELISA assays were performed in duplicate and triplicate, respectively. Data for zymographies and ELISA are expressed as change from the control condition (vehicle), $* P<0.05$ compared with vehicle.

kDa cutoff range for retention and then diluted it 1:100 in fresh DMEM/F12 culture medium. The reconstituted $\mathrm{CM}(\mathrm{RCM})$ were used in in vitro $3 \mathrm{D}$ angiogenesis assays. The results at 24 and $48 \mathrm{~h}$ showed that RCM from ESC stimulated with hCG (10 or $100 \mathrm{IU} / \mathrm{mL}$; RCM-10 and -100 , respectively) or vehicle (RCM), completely inhibited the sprouting of Ea.hy926 cells in microcarriers to levels below the control condition (Fig. 7A). The same effect was observed when hCG was added to RCM $(\mathrm{RCM}+\mathrm{hCG})$. The inhibition was also observed for VEGF (RCM+VEGFA); however, the reduction was less dramatic, reaching a $64.6 \%$ and $69.4 \%$ decrease at 24 and $48 \mathrm{~h}$, respectively (Fig. 7A).

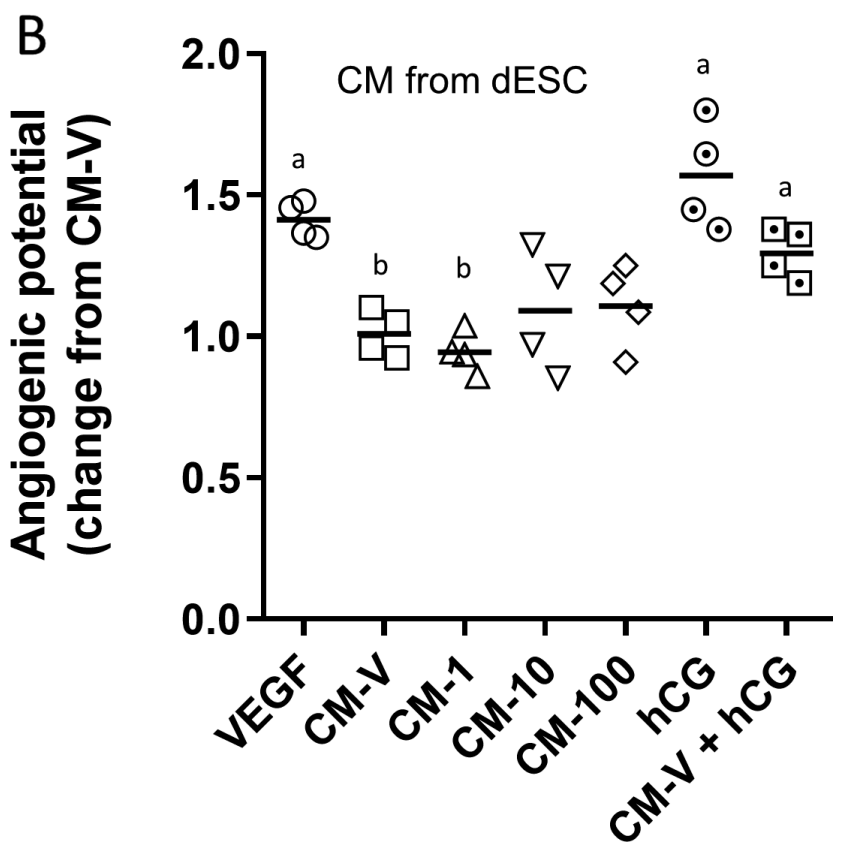

Figure 5 Angiogenic activity of conditioned media (CM) from human endometrial stromal cell (ESC) cultures in a 2D angiogenesis assay in vitro. Conditioned media $(\mathrm{CM})$ was prepared from human endometrial stromal cells (ESC, $n=4$ ) or in vitro decidualized ESC $(\mathrm{dESC}, n=4)$ stimulated for $48 \mathrm{~h}$ with vehicle $(\mathrm{CM}-\mathrm{V})$ or 1,10 or 100 $\mathrm{IU} / \mathrm{mL}$ of hCG (CM-1, CM-10 and CM-100, respectively).

Endothelium-derived EA.hy926 cells were seeded on Matrigel and incubated with the CM prepared from ESC (A) or dESC (B). EA.hy 926 cells were also incubated with $100 \mathrm{IU} / \mathrm{mL}$ hCG alone (hCG) or in combination with CM-V (CM-V+hCG) from ESC (A) and dESC (B). VEGF $(10 \mathrm{ng} / \mathrm{mL})$ was used as a positive control. EA.hy926 cells were photographed after $6 \mathrm{~h}$ of treatments using an inverted phase contrast photomicroscope. Data are expressed as change from CM-V. $n=4$, a $\neq \mathrm{b}, \mathrm{c} \neq \mathrm{d} ; P<0.05$. 

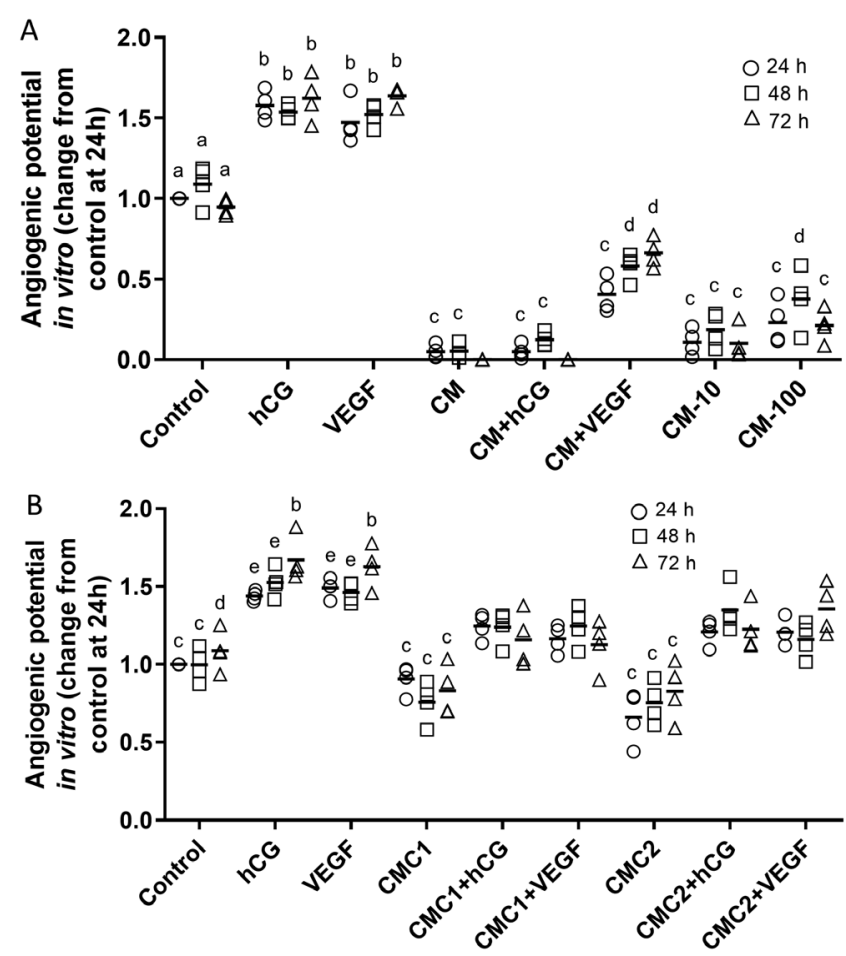

Figure 6 Angiogenic activity of conditioned media (CM) from cell cultures in a 3D angiogenesis assay in vitro at 24, 48 and $72 \mathrm{~h}$. (A) Microcarrier beads coated with EA.hy926 cells were embedded in a fibrin gel and then incubated with hCG (100 IU/mL), VEGFA (10 ng/ $\mathrm{mL}$ ) or CM from human endometrial stromal cell (ESC) cultures stimulated for $48 \mathrm{~h}$ with vehicle (CMV); or 10 or $100 \mathrm{IU} / \mathrm{mL}$ of hCG (CMV-10 and CMV-100, respectively). (B) Embedded microcarriers with EA.hy926 cells were also incubated in control CM prepared with NRK (kidney fibroblasts derived, CMC1) and TM4 (sertoli cells derived, CMC2) cell cultures in the presence or absence of hCG or VEGFA as previously. Data are presented as change from control at $24 \mathrm{~h}$ (non-treated cells). $n=4, \mathrm{~b} \neq \mathrm{a}, \mathrm{c}$ and $\mathrm{d} ; \mathrm{c} \neq \mathrm{a}$ and $\mathrm{e}, P<0.05$.

\section{hCG with or without CM from ESC or dESC do not change Eahy.296 cell viability}

Proliferation of endothelial cells is an important event that contributes to the angiogenesis process. We evaluated the effect of hCG, VEGFA and CM from ESC and dESC stimulated with hCG or vehicle on the viability of Eahy.296 cells. No significant differences were observed among all the assayed conditions, except for VEGFA that significantly increased cell viability in $50.4 \%$ compared with the control condition (Fig. 7B). This suggests that the regulatory effect of $\mathrm{CM}$ in angiogenesis is not mediated by a reduction in endothelial cell viability.

\section{ESC-derived CM inhibited cell migration in HUVEC cells}

To rule out that the antiangiogenic effect observed for ESC-derived CM on hCG, was specific for the endotheliallike cell line Ea.hy926, we performed a wound-healing in vitro assay to assess the effect of hCG on the migratory

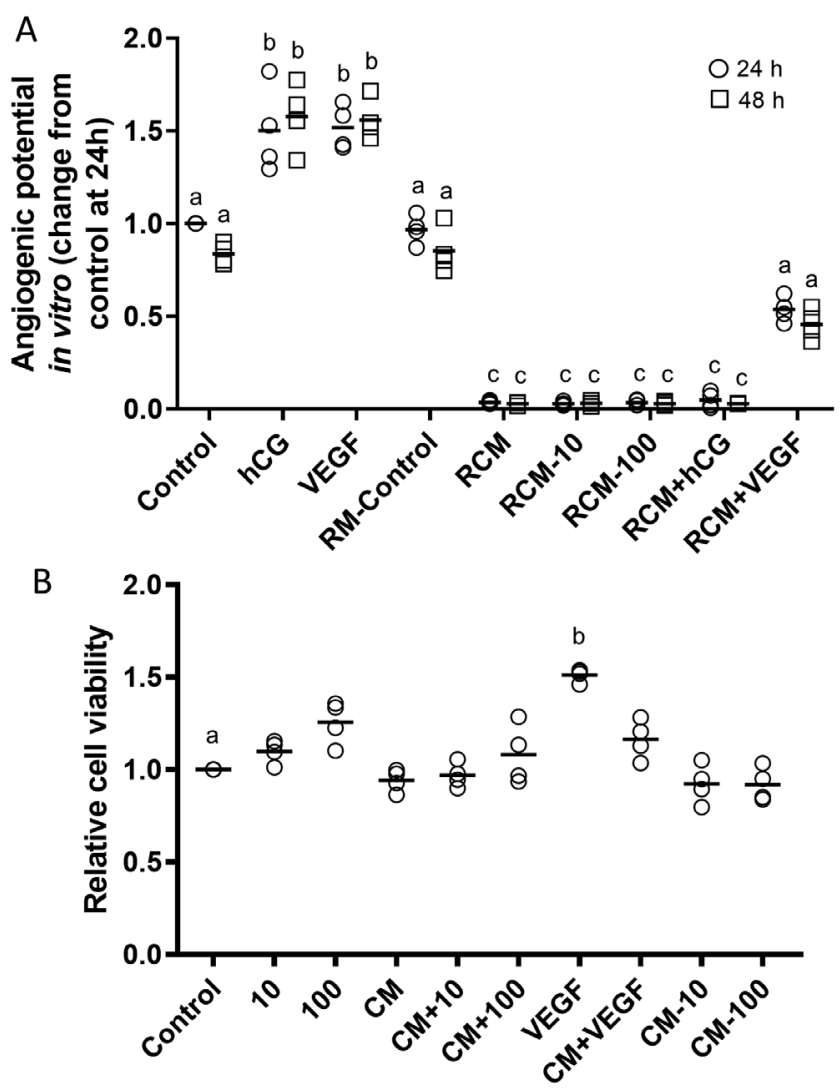

Figure 7 Secretions from endometrial stromal cells exert a regulatory effect on angiogenic potential in vitro related with sprout formation but do not affect viability of endothelial cells. (A) Angiogenic activity of reconstituted conditioned media (RCM) from endometrial stromal cell cultures in a 3D angiogenesis assay in vitro at 24, 48 and $72 \mathrm{~h}$. Microcarriers beads coated with EA.hy926 cells embedded in a fibrin gel were incubated with hCG $(100 \mathrm{IU} / \mathrm{mL})$, VEGFA $(10 \mathrm{ng} / \mathrm{mL})$ or RCM from stromal cells stimulated for $48 \mathrm{~h}$ with vehicle (RCM-V); or 10 or $100 \mathrm{IU} / \mathrm{mL}$ of hCG (RCM-10 and CM-100, respectively). RCM were prepared by concentration of $\mathrm{CM}(\times 100)$ with centrifugal filter devices and a $100 \times$ dilution with fresh culture medium. Control RCM (RCM-control) consisted on diluted concentratedunconditioned culture medium. CM from ESC induced a profound reduction in number of sprouts regardless of the previous hCG stimulus or in the presence of hCG or VEGF. (B) Cell viability of EA. hy 926 cells is not significantly modified by conditioned medium (CM) from endometrial stromal cell (ESC) cultures. Cell viability of EA.hy926 cells was assessed with an MTS assay after incubation for $24 \mathrm{~h}$ with hCG at 10 and $100 \mathrm{IU} / \mathrm{mL}$ (10 and 100, respectively), VEGFA $(10 \mathrm{ng} / \mathrm{mL})$ or CM from ESC stimulated for $48 \mathrm{~h}$ with vehicle (CM); or 10 or $100 \mathrm{IU} / \mathrm{mL}$ of hCG (CMV-10 and CMV-100, respectively). EA.hy926 cells were also incubated for $24 \mathrm{~h}$ with $\mathrm{CM}$ in the presence of 10 or $100 \mathrm{IU} / \mathrm{mL}$ of hCG $(C M+10$ and $\mathrm{CM}+100$, respectively) or $10 \mathrm{ng} / \mathrm{mL}$ of VEGFA (CM+VEGFA). Data are expressed as change from control (non-treated cells). $n=4, \mathrm{a} \neq \mathrm{b}$ and c, $P<0.05$.

behavior of HUVEC cells in the presence of ESC-derived CM. The wound-healing assay at 3 and $6 \mathrm{~h}$ demonstrated that HUVECs in the presence of CM exhibited a dramatic reduction in their motility than control cells, indicating that the spontaneous basal migratory behavior was 


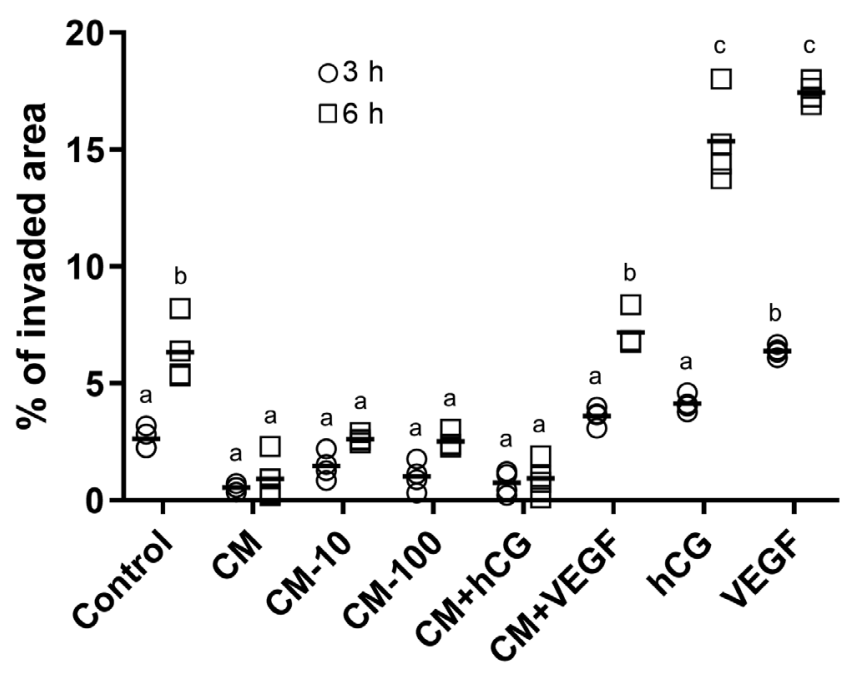

Figure 8 Conditioned media (CM) from human endometrial stromal cell (ESC) cultures reduce HUVEC migration in wound healing assay. (A) A bar graph plotting the relative efficiency of wound closure shows CM treatment significantly inhibited wound-closure when evaluated at 3 and $6 \mathrm{~h}$ after incubation with $\mathrm{CM}$ regardless of the previous hCG stimulus of ESC (CM-10 and CM-100) or in the presence of $100 \mathrm{IU} / \mathrm{mL}$ hCG $(\mathrm{CM}+\mathrm{hCG})$ reaching levels below the control condition. CM completely inhibited the stimulatory effect of hCG on HUVEC migration, whereas decreased to control values when co-incubated with VEGFA compared to VEGFA alone. Data are presented as the percentage of invaded area at 3 and $6 \mathrm{~h} ; \mathrm{a} \neq \mathrm{b}$ and $c ; c \neq b, P<0.05$.

restrained by secretions from ESC. Such profound cell motility inhibition was also observed with CM from ESC previously stimulated with hCG (10 or $100 \mathrm{IU} / \mathrm{mL})$. As expected, after $6 \mathrm{~h}$ hCG and VEGFA increased 2.4- and 2.7-fold, respectively, the migrated area by endothelial cells. CM from ESC, partially and almost completely blocked the pro-migratory effect induced by VEGFA and hCG, respectively $(-58.9 \%$ and $-93.8 \%$; Fig. 8$)$. Representative photomicrographs of the wound-healing in vitro assay are shown in Supplementary Fig. 4.

\section{Discussion}

In this study, we analyzed the regulation of 16 angiogenesis-related transcripts in the endometrium after intrauterine administration of hCG. Our results showed that transcript and protein levels for VEGFA and MMP3 increased. Although TIMP1 mRNA levels did not change, its protein levels decreased significantly. The immunostaining confirmed a strong up-regulation of VEGFA and MMP3 both endometrial epithelium and stroma, and reduced immunoreactive TIMP1 in line with the results from the Western blot analyses. A direct angiogenesis-promoting effect of hCG has been shown on endothelial cells through an agonistic effect on the LHCGR but also an indirect mechanism has been proposed inducing the secretion of VEGFA from the endometrial epithelium (Berndt et al. 2006) which may enhance blood vessel formation because a significant induction in theVEGFA secreted by the endometrium was reported upon intrauterine administration of hCG (Licht et al. 2007). We indeed observed that in the endometrial samples from women with hCG administration, VEGFA immunostaining in the uterine epithelia was stronger than in the stroma. Most of the upregulated VEGFA in the endometrial epithelium has been shown to be apically secreted to the uterine lumen (Hornung et al. 1998) which is supported by in vitro assays demonstrating that VEGFA is vectorially secreted by polarized endometrial epithelial cells through the apical domain (Taylor et al. 2001). Hence, such upregulated VEGFA in the epithelium may participate in early events of human implantation playing an embryotrophic role in the uterine lumen (Hannan et al. 2011) rather than regulating angiogenesis. The process of vascular expansion from pre-existent vessels is thought to be regulated by the balance of inducers and inhibitors within a given microenvironment (Iruela-Arispe \& Dvorak 1997). To gain further insights on the regulation of angiogenesisrelated molecules by hCG in the stromal endometrium, we focused on the role of the ESCs and dESC in regulating the angiogenic milieu during embryo implantation. ESC undergo decidualization during embryo implantation, which induce a profound morphological and biochemical changes that modify the secretory profile and potentially its response to hCG so we included in our experiments ESC and dESC. The screening for 55 secreted angiogenesis-regulating factors in cultured ESC and dESC stimulated with hCG did not show a clear proor anti-angiogenic response. The observed regulation of secreted TIMP1 in CM from ESC was unexpected based on the results obtained from the in vivo regulation and the data reported by our group showing a decrease of TIMP1 by ESC stimulated with hCG (Tapia-Pizarro et al. 2013). However, dose-response analyses showed a biphasic secretion of TIMP1, decreasing with $10 \mathrm{IU} /$ $\mathrm{mL}$ but increased with $100 \mathrm{IU} / \mathrm{mL}$. Such a response was not exhibited by dESC CM. In the in vivo study, VEGFA levels were significantly increased with the highest dose (5000 IU), whereas in the in vitro experiments, no differences were observed in ESC or dESC at the assayed doses $(1-100 \mathrm{IU} / \mathrm{mL})$. This apparently discordant result could be explained by several factors: the first one is that VEGFA induction is notably more pronounced in the epithelium than the underlying stroma as shown by the immunohistochemistry studies. Second, the dose and incubation times in both settings are different. Lastly, the endometrial tissue may contain several other cell types than ESC whose interplay may induce VEGFA in the stroma in response to hCG.

Angiogenesis is defined as the formation of new blood vessel growth from existent microvessels and involves several processes, including the proliferation of endothelial cells, proteolytic degradation of the extracellular matrix and migration of endothelial cells, 
leading to the organization of endothelial cells into capillary structures. In vitro, endothelial cells plated on a supporting matrix will invade the gel and selforganize into capillary-like structures depending on the surrounding stimuli (Vucenik et al. 2004). Using a 2D and a 3D approach for the evaluation of angiogenesis potential in vitro, we showed that ESC-derived CM disrupts the induction of in vitro tube formation by hCG. It has to be noted that secretions from ESC seem to have a greater inhibitory effect than dESC, likely due to a change in the secretory profile. Furthermore, our results showed that that the inhibition of the pro-angiogenic stimulus was partial for VEGFA, suggesting a specificity of the CM modulating the pro-angiogenic effect of hCG on endothelial cells. In addition, our results showed that ESC-secretions were particularly inhibitory of angiogenesis induced by hCG and VEGFA because CM derived from other cell cultures (TM4 or NRK) did not regulate with the same extension. Endothelial cell viability and migration are integral features of angiogenesis (Goodwin 2007). Although ESC-derived CM did not modify cell viability, migration assays showed that it significantly suppresses hCG-induced migratory potential of endothelial cells, suggesting that a migratory regulation is implicated as a mechanism for the inhibition of hCG-driven angiogenesis.

Zygmuntetal. (2002) proposed hCG as new angiogenic factor based on the direct effects of this hormone on endothelial cells. In 3D in vitro angiogenesis assays, hCG was shown to increase migration and formation of capillary-like structures by uterine endothelial cells. These observations were confirmed using ex vivo and in vivo assays showing a direct effect of hCG signaling through the LHCG receptor (LHCGR) in endothelial cells (Berndt et al. 2006). Taken together, the evidence suggested that upon pregnancy, hCG may induce uterine angiogenesis to support embryo implantation and placenta development. However, our results do not support a peri-trophoblastic angiostimulation directly mediated by hCG in the endometrial stroma. In fact, an anti-angiogenic effect was observed in $\mathrm{CM}$ derived from ESC or dESC, which is able to override the direct proangiogenic stimulus of hCG on the endothelial cells. The local modulation of direct pro-angiogenic effects of hCG could be exerted by ESC and dESC secretions, whereas during embryo implantation, other proangiogenic molecules secreted at the embryo-maternal interface (by trophoblast or uterine immune cells) such as VEGFA, may drive angiogenesis during embryo implantation. It has to be considered that the in vitro assays used in our study lack the biological complexity of human endometrial tissue. Leukocytes may constitute up to $25 \%$ of total cells in the uterine stroma during the secretory phase whose secretions may contribute to uterine angiogenesis. Uterine Natural Killer cells and macrophages are major sources of cytokines and angiogenic factors (Chen et al. 2017) so more studies should be performed using ex vivo and in vivo models to confirm our findings.

Our in vivo assays included women undergoing controlled ovarian stimulation for oocyte donation so the endometrial response to hCG may differ from a spontaneous ovulatory cycle. Our in vitro assays were performed using Eahy.296 and HUVEC as endothelial cells models, hence our results may differ in endometrial endothelial cells. However, it has been shown that several angiogenic molecules but not hCG induce angiogenesis in uterine microvascular cells (Kapiteijn et al. 2006).

Collectively, our data suggest that intrauterine administration of hCG does not induce an acute regulation of pro-angiogenesis molecules in the endometrial stroma in vivo and that ESC-secretions hinder the direct pro-angiogenic effects of hCG. ESC secretions seem to modulate endothelial cells migration; however, the specific secretory product eliciting this effect and the underlying mechanism by which it occurs is yet to be determined.

Overall, our results suggest that upon embryo implantation, the endometrial stroma may regulate the direct pro-angiogenic effect of hCG on endothelial cells so the local response toward promoting angiogenesis could be driven by other pro-angiogenic factors.

\section{Supplementary materials}

This is linked to the online version of the paper at https://doi. org/10.1530/REP-19-0559.

\section{Declaration of interest}

The authors declare that there is no conflict of interest that could be perceived as prejudicing the impartiality of the research reported.

\section{Funding}

FONDECYT 11100443, FONDECYT 1140614, PLISSER PCL0172012 and European Commission (REA) EU H2020MSCA-RISE-2015 grant 691058 MOMENDO.

\section{Author contribution statement}

A T-P, P C S and M R conceived and designed the experiments. $M R, B J, I N, F A, C V, D M-S$ and $M C J$ performed the experiments. A T-P, M R and M C J analyzed the data. P C S, E L A M, R G, M C J, A F and K S contributed to reagents/materials/ analysis tools/data interpretation. A T-P wrote the paper.

\section{Acknowledgements}

The authors would like to thank Sonia Dittus for assisting on the endometrial samples collection and Dr Soledad Henriquez for providing assistance of mounting and analyzing the 2D 
angiogenesis assays. The authors would like to thank also to the members of Prof Luigi Devoto lab at IDIMI.

\section{References}

Ahmed A, Dunk C, Ahmad S \& Khaliq A 2000 Regulation of placental vascular endothelial growth factor (VEGF) and placenta growth factor (PIGF) and soluble Flt-1 by oxygen - a review. Placenta 21 (Supplement A) S16-S24. (https://doi.org/10.1053/plac.1999.0524)

Aranda E \& Owen GI 2009 A semi-quantitative assay to screen for angiogenic compounds and compounds with angiogenic potential using the EA.hy926 endothelial cell line. Biological Research 42 377-389. (https://doi.org//S0716-97602009000300012)

Berndt S, Perrier d'Hauterive S, Blacher S, Péqueux C, Lorquet S, Munaut C, Applanat M, Hervé MA, Lamandé N, Corvol P et al. 2006 Angiogenic activity of human chorionic gonadotropin through $\mathrm{LH}$ receptor activation on endothelial and epithelial cells of the endometrium. FASEB Journal 20 2630-2632. (https://doi.org/10.1096/fj.06-5885fje)

Berndt S, Blacher S, Perrier d'Hauterive S, Thiry M, Tsampalas M, Cruz A, Péqueux C, Lorquet S, Munaut C, Noël A et al. 2009 Chorionic gonadotropin stimulation of angiogenesis and pericyte recruitment. Journal of Clinical Endocrinology and Metabolism 94 4567-4574. (https://doi.org/10.1210/jc.2009-0443)

Chen X, Man GCW, Liu Y, Wu F, Huang J, Li TC \& Wang CC 2017 Physiological and pathological angiogenesis in endometrium at the time of embryo implantation. American Journal of Reproductive Immunology 78 e12693. (https://doi.org/10.1111/aji.12693)

de Larco JE \& Todaro GJ 1978 Epithelioid and fibroblastic rat kidney cell clones: epidermal growth factor (EGF) receptors and the effect of mouse sarcoma virus transformation. Journal of Cellular Physiology 94 335-342. (https://doi.org/10.1002/jcp.1040940311)

Fazleabas AT, Kim JJ \& Strakova Z 2004 Implantation: embryonic signals and the modulation of the uterine environment - a review. Placenta 25 (Supplement A) S26-S31. (https://doi.org/10.1016/j.placenta.2004.01.014)

Fluhr H, Bischof-Islami D, Krenzer S, Licht P, Bischof P \& Zygmunt M 2008 Human chorionic gonadotropin stimulates matrix metalloproteinases-2 and -9 in cytotrophoblastic cells and decreases tissue inhibitor of metalloproteinases-1, -2 , and -3 in decidualized endometrial stromal cells. Fertility and Sterility 90 (Supplement) 1390-1395. (https://doi. org/10.1016/j.fertnstert.2007.08.023)

Goodwin AM 2007 In vitro assays of angiogenesis for assessment of angiogenic and anti-angiogenic agents. Microvascular Research $\mathbf{7 4}$ 172-183. (https://doi.org/10.1016/j.mvr.2007.05.006)

Hanahan D \& Folkman J 1996 Patterns and emerging mechanisms of the angiogenic switch during tumorigenesis. Cell 86 353-364. (https://doi. org/10.1016/s0092-8674(00)80108-7)

Hannan NJ, Paiva P, Meehan KL, Rombauts LJ, Gardner DK \& Salamonsen LA 2011 Analysis of fertility-related soluble mediators in human uterine fluid identifies VEGF as a key regulator of embryo implantation. Endocrinology 152 4948-4956. (https://doi.org/10.1210/ en.2011-1248)

Hornung D, Lebovic DI, Shifren JL, Vigne JL \& Taylor RN 1998 Vectorial secretion of vascular endothelial growth factor by polarized human endometrial epithelial cells. Fertility and Sterility 69 909-915. (https:// doi.org/10.1016/s0015-0282(98)00044-2)

Huang P, Wei L \& Li X 2017 A study of intrauterine infusion of human chorionic gonadotropin (hCG) before frozen-thawed embryo transfer after two or more implantation failures. Gynecological Endocrinology 33 67-69. (https://doi.org/10.1080/09513590.2016.1207164)

Iruela-Arispe ML \& Dvorak HF 1997 Angiogenesis: a dynamic balance of stimulators and inhibitors. Thrombosis and Haemostasis 78 672-677. (https://doi.org/10.1055/s-0038-1657610)

Kapiteijn K, Koolwijk P, van der Weiden RM, van Nieuw Amerongen G, Plaisier M, van Hinsbergh VW \& Helmerhorst FM 2006 Human embryoconditioned medium stimulates in vitro endometrial angiogenesis. Fertility and Sterility 85 (Supplement 1) 1232-1239. (https://doi. org/10.1016/j.fertnstert.2005.11.029)
Licht P, Fluhr H, Neuwinger J, Wallwiener D \& Wildt L 2007 Is human chorionic gonadotropin directly involved in the regulation of human implantation? Molecular and Cellular Endocrinology 269 85-92. (https:// doi.org/10.1016/j.mce.2006.09.016)

Licht P, Losch A, Dittrich R, Neuwinger J, Siebzehnrubl E \& Wildt L 1998 Novel insights into human endometrial paracrinology and embryo-maternal communication by intrauterine microdialysis. Human Reproduction Update 4 532-538. (https://doi.org/10.1093/ humupd/4.5.532)

Licht P, Russu V \&Wildt L 2001 On the role of human chorionic gonadotropin $(\mathrm{hCG})$ in the embryo-endometrial microenvironment: implications for differentiation and implantation. Seminars in Reproductive Medicine 19 37-47. (https://doi.org/10.1055/s-2001-13909)

Mansour R, Tawab N, Kamal O, El-Faissal Y, Serour A, Aboulghar M \& Serour G 2011 Intrauterine injection of human chorionic gonadotropin before embryo transfer significantly improves the implantation and pregnancy rates in in vitro fertilization/intracytoplasmic sperm injection: a prospective randomized study. Fertility and Sterility 96 1370.e1-1374. e1. (https://doi.org/10.1016/j.fertnstert.2011.09.044)

Mather JP 1980 Establishment and characterization of two distinct mouse testicular epithelial cell lines. Biology of Reproduction 23 243-252. (https://doi.org/10.1095/biolreprod23.1.243)

Minas V, Loutradis D \& Makrigiannakis A 2005 Factors controlling blastocyst implantation. Reproductive Biomedicine Online 10 205-216. (https://doi.org/10.1016/s1472-6483(10)60942-x)

Noyes RW, Hertig AT \& Rock J 1950 Dating the endometrial biopsy. Fertility and Sterility $13-25$. (https://doi.org/10.1016/S0015-0282(16)30062-0)

Perrier d'Hauterive S, Charlet-Renard C, Dubois M, Berndt S, Goffin F, Foidart JM \& Geenen V 2005 Human endometrial leukemia inhibitory factor and interleukin-6: control of secretion by transforming growth factor-beta-related members. Neuroimmunomodulation 12 157-163. (https://doi.org/10.1159/000084848)

Tapia-Pizarro A, Argandona F, Palomino WA \& Devoto L 2013 Human chorionic gonadotropin (hCG) modulation of TIMP1 secretion by human endometrial stromal cells facilitates extravillous trophoblast invasion in vitro. Human Reproduction 28 2215-2227. (https://doi.org/10.1093/ humrep/det136)

Taylor RN, Lebovic DI, Hornung D \& Mueller MD 2001 Endocrine and paracrine regulation of endometrial angiogenesis. Annals of the New York Academy of Sciences 943 109-121. (https://doi. org/10.1111/j.1749-6632.2001.tb03795.x)

Torry DS \& Torry RJ 1997 Angiogenesis and the expression of vascular endothelial growth factor in endometrium and placenta. American Journal of Reproductive Immunology $37 \quad 21-29 . \quad$ (https://doi. org/10.1111/j.1600-0897.1997.tb00189.x)

Toth P, Li X, Rao CV, Lincoln SR, Sanfilippo JS, Spinnato 2nd JA \& Yussman MA 1994 Expression of functional human chorionic gonadotropin/human luteinizing hormone receptor gene in human uterine arteries. Journal of Clinical Endocrinology and Metabolism 79 307-315. (https://doi.org/10.1210/jcem.79.1.8027246)

Vucenik I, Passaniti A, Vitolo MI, Tantivejkul K, Eggleton P \& Shamsuddin AM 2004 Anti-angiogenic activity of inositol hexaphosphate (IP6). Carcinogenesis 25 2115-2123. (https://doi.org/10.1093/carcin/ bgh232)

Zygmunt M, Herr F, Keller-Schoenwetter S, Kunzi-Rapp K, Munstedt K, Rao CV, Lang U \& Preissner KT 2002 Characterization of human chorionic gonadotropin as a novel angiogenic factor. Journal of Clinical Endocrinology and Metabolism 87 5290-5296. (https://doi.org/10.1210/ jc.2002-020642)

Received 21 November 2019

First decision 14 January 2020

Revised manuscript received 30 July 2020

Accepted 6 August 2020 